\title{
ON THE PERFORMANCE OF BASELINE SELF-CALIBRATION USING INTERSECTING INTERFEROMETRIC SAR ACQUISITIONS
}

\author{
Thomas Börner, Francesco De Zan, Paco López Dekker \\ German Aerospace Center (DLR), Microwaves and Radar Institute \\ ABSTRACT

\section{PHASE AND HEIGHT ERRORS}

\begin{abstract}
In this paper a baseline (height) self-calibration concept is introduced and applied to simulations of two different single-pass interferometric SAR systems with a singleswath and a dual-swath configuration. The results will show that the general performance is very promising and that, by simultaneously acquiring two separated swathes, accuracies in the sub-decimetric range can be reached.
\end{abstract}

Index Terms - Interferometry, SAR, calibration, performance, SIGNAL

\section{INTRODUCTION}

The following interferometric baseline self-calibration concept has been developed within the SIGNAL (SAR for Ice, Glacier aNd GlobAL Dynamics) Ka-band SAR mission study and some basic descriptions and results have already been reported in $[1,2,3]$. The need for such a self-calibration originated from the general problem of measuring the topography and topographical changes in large areas (Cryosphere) where the number of ground control points, usually required to get rid of systematic errors in the interferograms, is very low. Exactly this kind of problem was already addressed in studies for the Wide Swath Ocean Altimeter (WSOA) [4] and later on re-used in the Surface Water and Ocean Topography Mission (SWOT) concept [5], where platform roll uncertainties introduce interferometric errors, which are then reduced by crossover sampling techniques, leading to accuracies in the centimeter range. A very recent SWOT journal paper [8], which was published while this paper was written, is presenting the same theoretical approach and a similar calibration concept. In this paper, however, the performance will be estimated for different scenarios (or case studies) of a single-pass interferometric, side-looking SAR system observing snowand ice-covered landscapes (rather than an altimeter observing the ocean). In particular a traditional single-swath system will be compared to a new advanced dual-swath concept [3].

One of the main goals of the current self-calibration approach is to show that systematic errors can be dealt with even in the absence of stable control points.
The relative baseline between the two antennas is usually determined with high precision from double difference GPS measurements. However, there will remain errors in the millimeter range that will still affect topographic products (e.g. DEMs, Digital Elevation Maps). An error in the determination of the interferometric baseline will correspond to a phase error across the illuminated swath. When this phase error is converted to height (according to the phase-to-height coefficient) it will result into a height error. As an approximation, only small errors are considered that are in the orthogonal direction compared to the orientation of the across-track baseline. The errors parallel to the baseline don't play a significant role for small ground range values. The relative baseline error is thus approximately a rotation in the across track plane. The baseline error is then modeled as an autoregressive process with two poles (in the z-transform), symmetric with respect to the real axis. The closer the poles are to the unitary circle, the more coherent the process is over time. The phase of the poles corresponds to the orbital period, as observed and derived from very accurate along-track baseline measurements from the GRACE mission [6]. The calibration of these baseline errors would be easy if there were no additional error sources, e.g. the compared height will have errors due to coherence loss and other factors. On the other hand, the height itself could have changed between the two data takes, i.e. a physical height error (for example snowfall or melting processes) could be mistaken with a baseline-induced error. These height errors will be modeled using a multi-parameter statistical approach introducing height variance, spatial and temporal correlation. The challenge here is to find a simple model that is able to represent realistic (natural) effects, so that the performance evaluation of the calibration approach yields trustworthy results.

\section{SELF-CALIBRATION CONCEPT AND PERFORMANCE EVALUATION}

When an area is imaged by at least two orbits (one ascending and one descending) then we have an opportunity for calibration. The slopes induced by the two baseline 
errors are mostly uncorrelated and - more important - they have different characteristics. In particular the error vanishes at different points, since the two orbits will have different nadir projections on the ground. In the typical scenario of systematic SAR satellite mapping missions, mostly based on polar sun-synchronous orbits, the number of available intersections of ascending and descending data takes over an area of interest is quickly increasing within a reasonably short amount of time, particularly in areas above $\pm 60^{\circ}$ latitude. This opens the opportunity to use an increasing number of such intersections for a consecutive refinement of the calibration. However, there will be a limit of the refinement due to temporal decorrelation (strongly depending on local, daily and seasonal changes) and due to the spatial and temporal distribution of the intersections (depending on the instrument and orbital configuration). The geometry of calibration crossings is depicted in fig. 1.

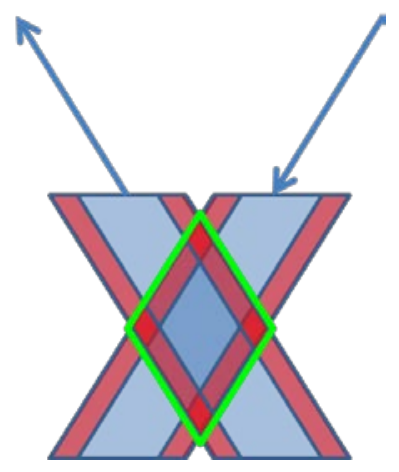

Figure 1: An ascending and a descending swath are crossing and thus build up a common diamond to be used for calibration. If a dual swath system (described in more detail in section 4) is used instead of a traditional single swath design, four small diamonds (red) are available.

For every orbital crossing we can write an equation like the following:

$$
\tilde{z}=k \cdot g r+z_{0}+z(t)
$$

The term $z_{0}$ represents the reference height, $k$ the baselineerror induced slope and $g r$ the ground range coordinate. The time-varying term $z(t)$ accounts for both physical height change and interferometric noise. When taking the differences between the heights measured from two different passes, the mean value of the height cancels out and is no more relevant. The time varying part will not, since some time will elapse between the passes. The slopeerror $k$ will also contribute to the measured height difference.

$$
\Delta \tilde{z}=k\left(t_{1}\right) g r_{1}-k\left(t_{0}\right) g r_{0}+\Delta z\left(t_{0}, t_{1}\right)
$$

Here $g r_{1}$ and $g r_{0}$ represent the far and near ground-range coordinates of the measured point from the two different passes. The two slopes $k$ are represented as a function of time. We will have one such equation for each calibration point (i.e. for each crossing where we will have two acquisitions). Collecting them in a system of equations and writing it in matrix form is immediate:

$$
\Delta \tilde{\mathbf{z}}=\mathbf{M k}+\Delta \mathbf{z}
$$

We need one more ingredient, which is the statistical characterization of the vector $\Delta \mathbf{z}$ in term of its covariance, i.e. the characterization of the correlations of height errors at two different points and at two different times. We assume that the process describing the height error is stationary, with zero mean and model it as a function of only three parameters:

1. one variance: $\sigma_{z}^{2}$

2. one parameter for spatial correlation: $\sigma_{d}^{2}$

3. one parameter for temporal correlation: $\tau$

Here is the adopted model for the spatial and temporal correlation:

$$
\begin{aligned}
& E\left[z^{(n)}\left(t_{a}\right) z^{(m)}\left(t_{b}\right)\right]= \\
& =\sigma_{z}^{2} \exp \left(-\left|t_{a}-t_{b}\right| / \tau\right) \cdot \mathrm{e}^{\left(-|d(m, n)|^{2} /\left(2 \cdot \sigma_{d}^{2}\right)\right)}
\end{aligned}
$$

where $d(m, n)$ is the distance between crossing $(m)$ and crossing $(n)$. This expression describes an exponential correlation along the time dimension, and a Gaussian correlation in the spatial dimension.

For the evaluation of the baseline calibration performance a Linear Minimum Mean Square Error (LMMSE) estimator of vector $\mathbf{k}$ is defined as an optimal linear combination of the height change observations $\Delta \tilde{\mathbf{z}}$, i.e.

$$
\hat{\mathbf{k}}=\mathbf{A} \mathbf{\Delta} \tilde{\mathbf{z}}
$$

where the matrix $\mathbf{A}$ is built so that the mean square error is minimized in a statistical sense. For the purpose of deriving the performance it is not necessary to write the explicit expression for $\mathbf{A}$, because the final performance is provided by the a posteriori covariance matrix $\mathbf{R}_{\hat{k}}$ of the vector $\hat{\mathbf{k}}$, which can be written as [7]:

$$
\mathbf{R}_{\hat{k}}=\left[\mathbf{R}_{k}^{-1}+\mathbf{M}^{T} \mathbf{R}_{z}^{-1} \mathbf{M}\right]^{-1}
$$

This expression can be interpreted saying that the a posteriori information on the baseline error (the groundrange slope) is equal to the a priori information $\mathbf{R}_{k}$ (derived 
from the autoregressive model) plus the noise information projected onto the baseline error space.

In a real application the performance might be worse or even better depending on the actual orbit accuracy, the statistical behavior of the topographical changes in space and time, and the available data of crossings.

\section{PERFORMANCE EVALUATION FOR SINGLE- AND DUAL-SWATH SCENARIOS}

In the SIGNAL mission concept study the performance of a particular scenario has already been evaluated [1]. Recently a more advanced instrument concept with a dual-swath architecture has been published [3] (a sketch of this concept is presented in fig. 2), i.e. acquiring two separated swathes simultaneously. The main advantage of this new concept is that the two swathes build up an effectively larger calibration diamond (see fig. 1) with a higher separation of near- and far-range information. According to the calibration concept described in section 3, a better performance can be expected, since it is mainly dependent on the difference between $g r_{0}$ and $g r_{1}$ (see eq. (2)).

For the following simulations a sun-synchronous dusk-dawn orbit with an above-ground height of $735 \mathrm{~km}$ and a repeat period of 11 days (159 revolutions) has been chosen. The single-swath is defined by the near-range and far-range look-angles of $29.6^{\circ}$ and $30.7^{\circ}$, respectively, leading to a swath width of $\sim 21 \mathrm{~km}$. The first swath of the dual-swath design is defined by look-angles of $29.9^{\circ}-31.2^{\circ}$, the second swath by 33.7-34.9, yielding two $\sim 25 \mathrm{~km}$ swathes separated by a gap of $\sim 50 \mathrm{~km}$. A baseline of $100 \mathrm{~m}$ has been simulated for both cases. The characterization of the correlations of height errors at different points and different times as described in eq. (4) may be defined in a first approach as $\sigma_{z}=1 \mathrm{~m}, \sigma_{d}=200 \mathrm{~km}$ and $\tau=10$ days.

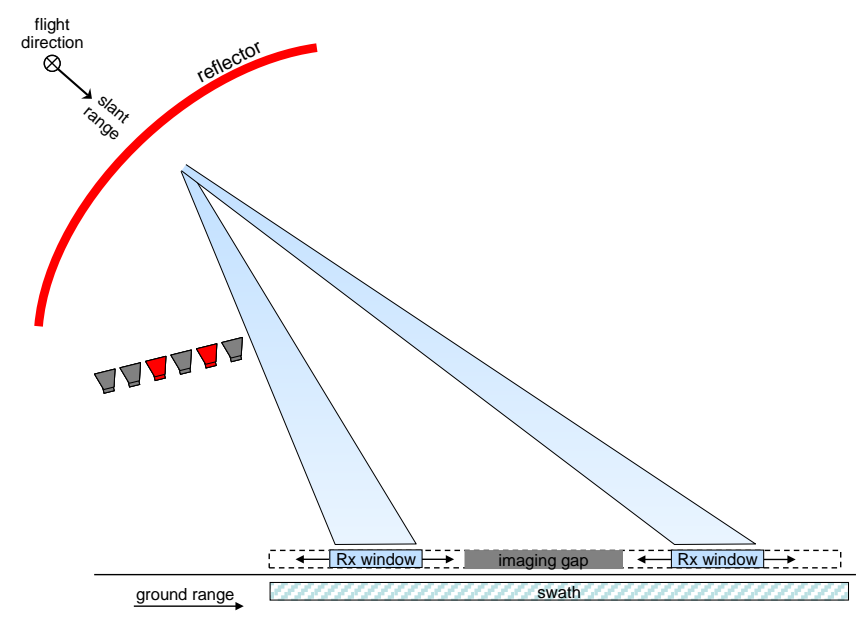

Figure 2: Schematic representation of the simultaneous dual-swath operation of a SAR instrument.
Since SIGNAL is a mission dedicated to the Cryosphere and since this kind of calibration is mainly required for areas without a sufficient number of ground control points, Greenland has been chosen as the region of interest, and the simulation results are restricted to potential crossings in this area only. Also the simulated time span is limited to the 11 day repeat period.

All available calibration diamonds over Greenland within 11 days are shown in fig. 3 (a) for the single-swath configuration, and in fig. 3 (b) for the dual-swath configuration, respectively. In order to calculate the performance of the calibration, one descending track has been selected, represented by the blue line, for which the residual height error after calibration is calculated (see fig. 3 (c). The dual-swath configuration clearly outperforms the single-swath, as expected from the larger diamonds. However, the biggest difference is observed at around $72^{\circ}$ latitude, whereas for higher latitudes the dual-swath height error increases again and comes close to the single-swath height error. This behavior can be explained by the increasing density of crossings at higher latitudes and the fact that the Greenland area restricts the number of crossings that can be used for calibration. Since more small diamonds fit into the same area, the single-swath configuration can compensate for the lack of diamond size. Therefore it is of interest what happens if the number of diamonds that can be used for calibration is reduced. To simulate this, a so-called thinning factor is introduced. A factor of 1 means that all available diamonds are used, a factor of 2 means that only every second is chosen for calibration, 3 - every third, etc. As can be derived from fig. 3 (d), the performance is strongly dependent on the selection of available diamonds. By applying the thinning factor, no intelligence is used to make a good choice, so that the performance of the dual-swath can become as bad as the single-swath one for some particular cases.

\section{CONCLUSIONS}

A performance evaluation of a height self-calibration concept for two sensor configurations (single- and dualswath) has been carried out over a selected scenario over Greenland. The average residual error for the single-swath configuration is around $20 \mathrm{~cm}$, whereas the dual-swath yields an average of around $10 \mathrm{~cm}$ (but may go down below $5 \mathrm{~cm}$ under ideal conditions). In general the performance goes down at the edges of the area of interest due to the geometrical limitations for the calibration diamonds. The authors believe there is still a lot of room for improvement by exploiting data sets over a longer observation time (many consecutive repeat cycles) and by a more sophisticated selection of calibration diamonds. Thus the next step is to evaluate a full 2-D performance (all available tracks) with a longer time span. 


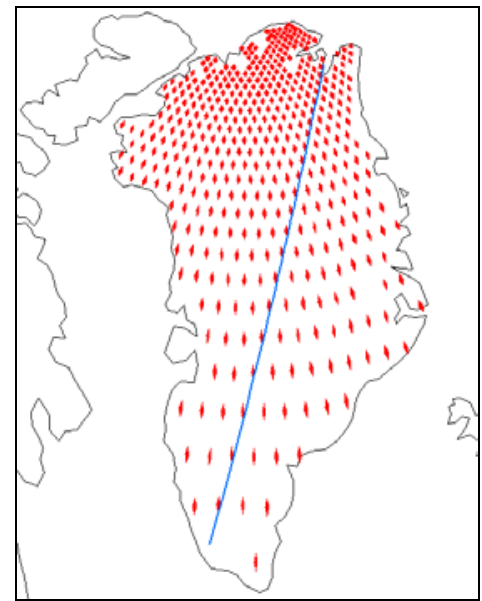

(a)

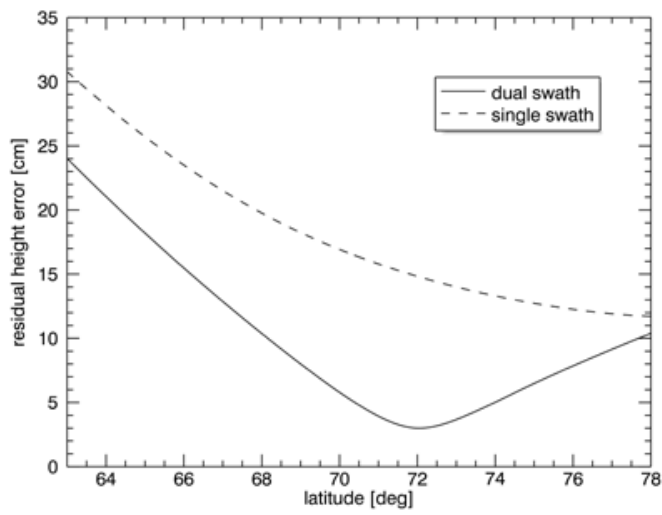

(c)

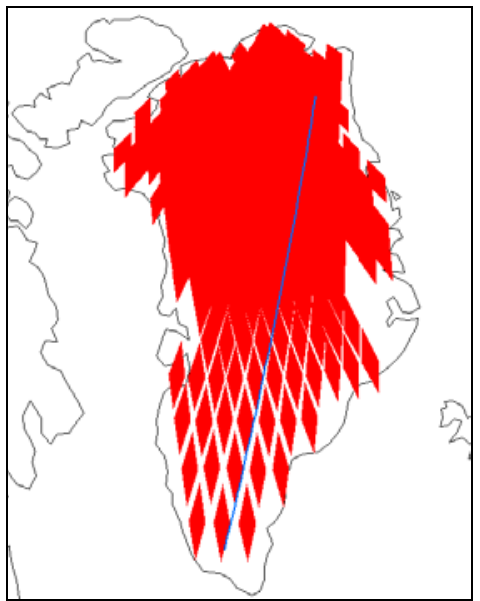

(b)

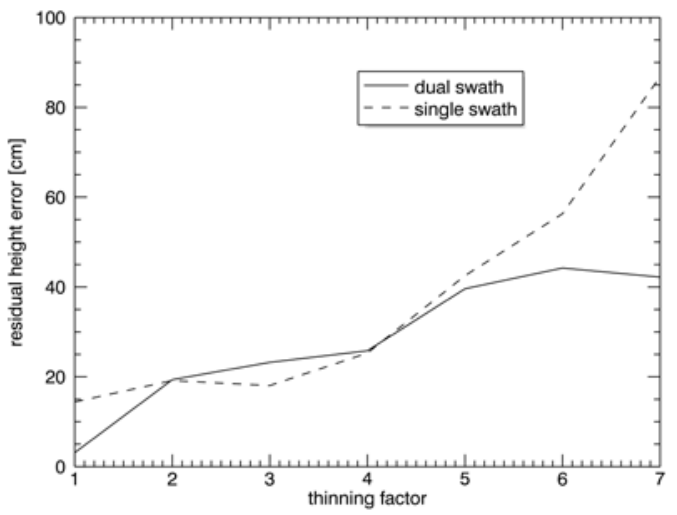

(d)

Figure 3: Maps of crossings over Greenland within an 11 day repeat cycle, and performance results. (a) Single-swath crossings - the blue line denotes the selected descending track to be calibrated. (b) Dual-swath crossings. (c) Residual height error vs. latitude. (d) Residual height error at $72^{\circ}$ latitude vs. thinning factor.

\section{REFERENCES}

[1] T. Börner, F. De Zan, P. López-Dekker, G. Krieger, I. Hajnsek, K. Papathanassiou, M. Villano, M. Younis, A. Danklmayer, W. Dierking, T. Nagler, H. Rott, S. Lehner, T. Fügen, and A. Moreira., "SIGNAL : SAR for Ice, Glacier and Global Dynamics," Proc. IGARSS 2010, Geoscience and Remote Sensing Symposium (IGARSS) 2010, Honolulu, Hawaii, pp. 2884-2887, 25-30 July 2010.

[2] M. Villano, A. Moreira, H. Miller, H. Rott, I. Hajnsek, R. Bamler, P. López-Dekker, T. Börner, F. De Zan, G. Krieger, K. Papathanassiou, "SIGNAL: Mission Concept and Performance Assessment," Proc. EUSAR Conference 2010, pp. 520-523, Aachen, Germany, 08-10 June 2010.

[3] P. López-Dekker, M. Younis, T. Börner, and G. Krieger, "SIGNAL: A Ka-band Digital Beam-Forming SAR System Concept to Monitor Topography Variations of Ice Caps and Glaciers,” Proc. ARSI Conference 2011, ESA ESTEC, Noordwijk, Netherlands, 13-15 September 2011.
[4] E. Rodriguez, B. Pollard, "Centimetric sea surface height accuracy using the Wide-Swath Ocean altimeter," Proc. IGARSS 2003, Toulouse, France, pp. 3011-3013, vol. 5, 21-25 July 2003.

[5] M. Durand, L. Fu, D.P. Lettenmaier, D.E. Alsdorf, E. Rodriguez, D. Esteban-Fernandez, "The Surface Water and Ocean Topography Mission: Observing Terrestrial Surface Water and Oceanic Submesoscale Eddies,” Proc. IEEE, vol. 98, issue 5, pp. 766-779, May 2010.

[6] J. Hueso Gonzáles et al., "Development of the TanDEM-X Calibration Concept: Analysis of Systematic Errors”, IEEE Trans. Geosci. Remote Sensing, vol. 48 (2), 2010.

[7] S. Kay, "Fundamentals of statistical signal processing: Estimation theory,” Prentice Hall, 1993.

[8] G. Dibarboure, S. Labroue, M. Ablain, R. Fjørtoft, A. Mallet, J. Lambin, and J. Souyris, "Empirical Cross-Calibration of Coherent SWOT Errors Using External References and the Altimetry Constellation,” IEEE Trans. Geosc. Remote Sensing, vol. 50, no. 6, June 2012. 